\title{
Estructura Factorial, Invarianza Métrica y Propiedades Psicométricas de la Versión Chilena del Cuestionario de Perdón a la Ex Pareja
}

\author{
Factorial Structure, Metric Invariance and Psychometric Properties of the Chilean \\ Version of the Questionnaire of Forgiveness of the Former Partner
}

\author{
Mónica Guzmán-González ${ }^{1}$, Diana Rivera-Ottenberger ${ }^{2}$, Lusmenia Garrido $^{3}$, Paula Contreras $^{4}$ y \\ Sagrario Yárnoz-Yaben ${ }^{5}$
}

\begin{abstract}
Resumen
El presente estudio tuvo como objetivo evaluar la estructura factorial de la versión Chilena del Cuestionario de perdón a la ex pareja (CPD-S; Yárnoz-Yaben \& Comino, 2012), examinar la invarianza métrica según sexo y tipo de relación (matrimonios y convivencias), estimar la consistencia interna de la escala y evaluar su asociación con indicadores de salud mental y satisfacción con la vida. Para ello, se evaluó una muestra de 715 personas separadas o divorciadas chilenas. Los datos proporcionan evidencia que el instrumento se ajusta aceptablemente a una estructura unidimensional, que la escala es invariante según sexo y tipo de relación y que posee una consistencia interna adecuada. Además, se identificaron asociaciones significativas con medidas de salud mental y satisfacción con la vida en las direcciones esperadas. En conclusión, se sostiene la validez de las puntuaciones del CPD-S para su uso en contexto chileno.
\end{abstract}

Palabras clave: Cuestionario Perdón a ex pareja, propiedades psicométricas, invarianza métrica, personas divorciadas

\begin{abstract}
The objective of this study was to evaluate the factorial structure of the Chilean version of the Questionnaire of Forgiveness of the Former Partner (CPD-S; Yárnoz-Yaben \& Comino, 2012) to examine its metric equivalence according to sex and type of relationship (marriages and cohabitation), to evaluate its association with mental health indicators and life satisfaction and estimate its internal consistency. For this, a sample of 715 separated or divorced Chilean people was evaluated. Data provided evidence that the instrument fits properly to an one-dimensional structure, that the scale is invariant according to sex and type of relationship and that it has an adequate internal consistency. In addition, significant associations were identified with measures of mental health and life satisfaction in the expected directions. In conclusion, the validity of the CPD-S scores for its use in the Chilean context is supported.
\end{abstract}

Keywords: Questionnaire of Forgiveness of the Former Partner, psychometric properties, metric invariance, divorced people

Esta investigación ha sido financiada por el Fondo Nacional de Ciencia y Tecnología del Gobierno de Chile (Fondecyt 1150044), concedido a la primera autora.

${ }^{1}$ Doctora en Psicología. Escuela de Psicología, Universidad Católica del Norte, Chile. Profesora Titular. Av. Angamos 0610, Antofagasta, Región de Antofagasta, Chile. Tel: +56552355822. Correo: moguzman@ucn.cl

${ }^{2}$ Doctora en Psicología. Escuela de Psicología, Pontificia Universidad Católica de Chile. Profesora Asociada. Av. Vicuña Mackenna 4860, Macul, Santiago, Chile. Tel: +56223544609. Correo: dvrivera@uc.cl

${ }^{3}$ Magister en Psicología Clínica. Departamento de Psicología, Universidad Católica del Maule. Profesora Auxiliar. Avenida San

Miguel 3605. Talca, Chile. Tel: +56712203139. Correo: lgarrido@ucm.cl

${ }^{4}$ Máster en Terapia Cognitivo Social, Universidad de Barcelona. Escuela de Psicología, Universidad Católica del Norte, Chile. Profesora Asociada Av. Angamos 0610, Antofagasta, Región de Antofagasta, Chile. Teléfono: +56 55 2355822. Correo: pcontreras@ucn.cl

5 Doctora en Psicología. Departamento de Personalidad, Evaluación y Tratamientos Psicológicos, Facultad de Psicología, Universidad del País Vasco (UPV-EHU), España. Profesora Titular. Avenida Tolosa Etorbidea 70, 20018 Donostia-San Sebastián, España. Teléfono: 943018346 Fax: 943 015670. Correo: sagrario.yarnoz@ehu.eus 


\section{Introducción}

El divorcio y la separación constituyen fenómenos frecuentes, que suelen acompañarse de un amplio rango de reacciones emocionales, que van desde el alivio cuando la separación implica la salida de una relación insatisfactoria (Tashiro, Frazier \& Berman, 2006), hasta intenso sufrimiento y malestar en otros casos (Sbarra, Hasselmo, \& Bourassa, 2015; Sbarra, 2015). La intensidad de los sentimientos y vivencias experimentadas ha hecho que la ruptura de la relación se asemeje a o conceptualice como un proceso de duelo (Sánchez-Aragón \& RetanaFranco, 2013).

En lo que respecta a la separación de personas casadas, a nivel internacional se ha reportado que el divorcio es un fenómeno frecuente (Amato, 2010), siendo una realidad de la que Chile no se encuentra exenta (United Nations, 2011). A tales rupturas, se suma el quiebre de uniones de hecho, forma de vinculación que ha aumentado en los últimos años, cuya significación social ha ido cambiando hacia una paulatina validación de nuevas organizaciones familiares (Berrington, Perelli-Harris, \& Trevena, 2015; Ojeda, Torres, \& Moreira, 2010; Svenson \& Siles, 2014), pero que ha sido largamente excluida en los estudios sobre los efectos de la separación.

La transición a una nueva etapa asociada a la separación, involucra el enfrentamiento de una serie de cambios importantes en diversas esferas de la vida, como la reorganización de la estructura familiar y sus roles, cambios en las redes de apoyo y de las condiciones económicas, entre otros (Cáceres, Manhey, \& Raies, 2004). Asimismo, el término de una relación de pareja implica un proceso de adaptación psicológica y el afrontamiento de una serie de desafíos específicos, como son el desapego de la ex pareja, el manejo de las emociones asociadas a la pérdida y la redefinición de la propia identidad (Sweeper \& Halford, 2006).

En el marco de la comprensión de la adaptación a la separación, diversos autores han planteado la importancia del perdón a la ex pareja, dado que se relacionaría con mayor bienestar psicológico, mejor adaptación a la separación, mayor satisfacción con la vida, y menores índices de problemas de salud mental post ruptura (e.g.,
Bonach \& Sales, 2002; 2007; 2009; Rohde-Brown \& Rudestam, 2011; Rye, Folck, Heim, Olszewski, \& Traina, 2004; Yárnoz-Yaben, 2013).

Asimismo, diversos estudios han reportado que el resentimiento hacia la ex pareja, la hostilidad y el deseo de castigar al otro, expresado muchas veces a través del entorpecimiento de las relaciones con los hijos, trae consecuencias negativas sobre estos (Trinder, Kellet, \& Swift, 2008), siendo uno de los aspectos que caracteriza a los divorcios altamente conflictivos (Fabricius \& Luecken, 2007; Lebow \& Newcombrekart, 2007). En ese marco, el perdón a la ex pareja se ha asociado a una alianza coparental más positiva (Bonach, 2007, 2009; Bonach \& Sales, 2002) y menores consecuencias desfavorables sobre los hijos (Freedman \& Knupp, 2003).

Tales antecedentes fundamentan la importancia de estudiar el perdón como un aspecto importante en la dinámica y adaptación post separación, y han contribuido al desarrollo de intervenciones que tienen como propósito facilitar el perdón a la ex pareja para el logro de una coparentalidad más positiva (e.g., Bonach, 2007; Rye et al., 2004; Rye et al., 2012).

Pese a que el tema de la adaptación a la separación y de factores asociados, ha sido ampliamente investigado en el ámbito internacional, la cantidad de estudios empíricos desarrollados en el contexto latinoamericano y chileno en particular son todavía escasos, lo que no permite comprender, por ejemplo, las variaciones culturales asociadas a cómo se vive este proceso, en un lugar como Chile, con tradición más bien conservadora, que tiene la particularidad de haber sido uno de los últimos países a nivel mundial en legalizar el divorcio, el año 2004. En tal sentido, un aspecto pendiente es contar con una batería de instrumentos adaptados al contexto local que permitan iniciar y consolidar una línea de investigación en este ámbito, siendo una de ellas el estudio del perdón a la ex pareja, como factor relevante asociado a la adaptación a la separación. Es sobre este aspecto que el presente estudio pretende aportar.

El perdón interpersonal puede ser entendido como una estrategia de afrontamiento que implica la neutralización de un estresor que es resultado de la percepción de una herida u ofensa interpersonal (Strelan \& Covic, 2006). El perdón 
involucra un conjunto de respuestas cognitivas, afectivas y, conductuales ante una trasgresión, que implican la disminución de sentimientos negativos (resentimiento y enojo) hacia el ofensor/a, y un menor deseo de castigarlo/a (Gordon \& Baucom, 2003; Rye et al., 2014; Yárnoz-Yaben \& Comino, 2012).

El perdón difiere de conceptos relacionados como el olvido, la reconciliación o la negación de la ofensa, siendo más bien una decisión y un estado que se alcanza reconociendo la naturaleza hiriente de la transgresión, sin que necesariamente involucre el restablecimiento de la relación (Guzmán, 2010).

Para la medición del perdón existe en la actualidad una variedad considerable de instrumentos, tales como el Enright Forgiveness Inventory (EFI; Subkobiak et al., 1995), la Forgiveness Scale (FS; Rye et al., 2001), la Heartland Forgiveness Scale (HFS; Thompson et al., 2005), el Transgression Narrative Test of Forgiveness (TNTF; Berry, Worthington, Parrott, O'Connor, \& Wade, 2001), el Marital Offencespecific Forgiveness Scale (MOFS; Paleari, Regalia, \& Fincham, 2009) y el Transgression Related Interpersonal Motivations (TRIM; McCullough, et al., 1998; McCullough, Root, \& Cohen, 2006).

Los instrumentos antes descritos tienen como objetivo medir el perdón episódico, es decir, de una ofensa específica (e.g., TRIM) o bien disposicional, como una tendencia general o una característica de personalidad estable en el tiempo (e.g., HFS), salvo el MOFS, diseñado específicamente para evaluar el perdón en relaciones amorosas. Sin embargo, los instrumentos orientados a medir el perdón en el contexto específico del divorcio y la separación son escasos, salvo el Cuestionario de Perdón en el Divorcio y la Separación (CPD-S), elaborado en España por Yárnoz-Yaben y Comino (2012), que es el objeto de análisis del presente estudio.

El CPD-S fue desarrollado a partir de la revisión de ítems de una serie de instrumentos que cubrían aspectos del perdón susceptibles de ser aplicados en la situación de divorcio y separación. De un conjunto de 15 ítems iniciales, se retuvieron 5, que cubren aspectos como el rencor, la rabia, la culpabilización del otro, y la compasión. Como resultado, se obtuvo una escala breve, de fácil respuesta y corrección. En cuanto a sus propiedades psicométricas, se retuvo una solución unifactorial mediante un análisis de componentes principales, y el índice de fiabilidad obtenido mediante el alpha de Cronbach fue de .077. Además, se aportó evidencia de validez convergente y discriminante a partir de la asociación de los puntajes del CPD-S con medidas de adaptación a la separación, satisfacción con la vida, disposición a la coparentalidad y presencia de problemas de conducta en los hijos (YárnozYaben \& Comino, 2012).

Pese a que el CPD-S ya se encuentra en castellano, la validación del uso de las puntuaciones de esta escala en otros contextos es necesaria, dadas las diferencias lingüísticas y culturales que pueden detectarse aun cuando el idioma sea el mismo (Hambleton, Merenda, \& Spielberg, 2005).

Además del examen de la dimensionalidad del CPD-S en contexto chileno, se busca, en el presente estudio, revalidar los hallazgos reportados por Yárnoz-Yaben y Comino (2012) sobre la estructura factorial, ocupando una técnica de análisis más potente estadísticamente y que controla el error de medida, como es el análisis factorial confirmatorio, lo que no es posible con el uso de análisis de componentes principales.

Adicionalmente, es de relevancia identificar si la escala se comporta de manera similar para hombres y mujeres, dado que las comparaciones por género en constructos psicológicos latentes como el perdón son frecuentes en la literatura, pero un paso previo es comprobar si un instrumento funciona de modo equivalente en los grupos que se desea contrastar. En la misma línea, se busca evaluar si el CPD-S funciona igual en personas provenientes de relaciones de matrimonio y relaciones de convivencia, dado que la mayor parte de los estudios en el contexto del divorcio y la separación han incluido solo personas provenientes de uniones legales. La exclusión de personas que sostuvieron una relación de convivencia en los estudios sobre adaptación a la separación, deja fuera la posibilidad de comprender lo que sucede en una forma de vinculación que ha aumentado notoriamente en los últimos años.

En consecuencia, el objetivo de este estudio fue examinar la estructura factorial del CPD-S en 
contexto chileno, y evaluar la invarianza métrica de la escala según sexo y tipo de relación.

Secundariamente, se estimó la consistencia interna de la escala y se examinó la validez convergente de la versión chilena del CPD-S, mediante su asociación con medidas de salud mental y satisfacción con la vida.

A partir de los antecedentes previos, se hipotetizó que el modelo que evidenciaría un mejor ajuste a los datos será uno de carácter unidimensional y que el CPD-S será equivalente tanto para hombres y mujeres, y como para personas separadas provenientes de matrimonios y de uniones de hecho. También, que el perdón a la ex pareja se asociaría a mejores niveles de satisfacción vital y menores problemas de salud mental.

\section{Método}

\section{Participantes}

Este estudio formó parte de un proyecto más amplio cuyo objetivo fue evaluar los factores asociados al ajuste a la separación en adultos chilenos. Para ello, se evaluó una muestra no probabilística, balanceada por sexo y edad, compuesta por personas provenientes principalmente de las ciudades de Santiago, Antofagasta, Talca y Concepción. Los criterios de inclusión fueron ser mayor de 18 años y que al momento de la evaluación estuviesen separados o divorciados de una relación de pareja que hubiese durado al menos 1 año.

Se evaluaron dos muestras:

Muestra 1. Conformada por 237 participantes, distribuidos en 116 hombres (48.9\%) y 121 mujeres $(51.1 \%)$, con una edad promedio de 37.95 años $(D T=13.19)$ y un rango entre los 21 y los 65 años. Un $33.3 \%$ tenía estudios superiores, un $56.1 \%$ estudios medios o técnicos incompletos y el $10.6 \%$ tenía estudios básicos completos o menores. La ruptura había ocurrido en promedio hace 3.89 años $(D T=3.09)$.

Muestra 2. La muestra inicial estuvo compuesta por 741 personas. La inspección preliminar de los datos reveló la existencia de 21 casos con datos perdidos. Mediante prueba t y chi cuadrado, se identificó que no había diferencias estadísticamente significativas en variables sociodemográficas y propias de la separación (edad, sexo, nivel educacional, tipo de relación, presencia de hijos en común, duración de la relación, tiempo transcurrido desde la separación), entre quienes completaron todas las preguntas y quienes no lo hicieron. Adicionalmente, la prueba de aleatoriedad MCAR Little, dio por resultado un valor de Chi cuadrado=39.07 $(\mathrm{gl}=46 ; \mathrm{p}<.756)$, lo que indica que no tienen un patrón definido, y que son datos faltantes al azar. Con base en estos resultados, dichos casos fueron eliminados.

Asimismo, se identificó, mediante la distancia de Mahalanobis, la existencia de 5 casos atípicos multivariados que superaban el valor crítico $(p<.001)$, los cuales fueron removidos de la muestra (Meyers, Gamst, \& Guarino, 2013).

De este modo, se trabajó con datos completos y la muestra final quedó constituida por 715 personas, 358 mujeres $(50.1 \%)$ y 357 hombres (49.9\%), con edades entre los 21 y 69 años y un promedio de edad de 40.03 años $(D T=11.50)$. De ellos, 405 provenían de relaciones de matrimonio (56.6\%), y 310 de uniones de hecho (43.4\%).

Respecto de la distribución según nivel educacional, el porcentaje más alto de las personas reportó tener educación superior completa $(39 \%)$, seguida de educación superior incompleta (25.3\%), educación secundaria completa $(24.7 \%)$, mientras que el $11 \%$ tenía un estudios secundarios incompletos o menos.

En cuanto a la caracterización sobre el proceso de divorcio o separación, el tiempo promedio transcurrido desde la separación fue de 2.86 años $(D T=1.78)$. Un $43.6 \%$ señaló que la separación fue por iniciativa propia, un $25.8 \%$ reportó que fue iniciada por la ex pareja, mientras que el $30.6 \%$ restante indicó que fue una decisión compartida. Además, un $61.3 \%$ de los participantes señaló la presencia de hijos en común con la ex pareja.

El tiempo promedio de duración de la relación fue de 11.55 años $(D T=9.34)$, con una mediana de 8.08 años. Finalmente, un $38.3 \%$ de las personas señaló tener una nueva pareja.

\section{Procedimiento}

El estudio contó con la aprobación del comité de ética de las instituciones involucradas.

El proceso de adaptación del CPD-S se organizó en tres etapas. Inicialmente se realizó la 
adaptación lingüística de los ítems del CPD-S al contexto chileno, para lo cual 3 jueces expertos en psicología clínica y medición revisaron independientemente los reactivos de la escala. Luego elaboraron una versión consensuada del mismo.

En un segundo momento, la versión chilena fue aplicada a la primera muestra, para explorar la estructura factorial del CPD-S.

Finalmente, en una tercera fase, se aplicó la escala en la segunda muestra a fin de confirmar la estructura identificada en la primera etapa, además de examinar su consistencia interna y la validez concurrente.

Para el acceso a la muestra, se entrenaron 59 encuestadores en cuatro ciudades de Chile, quienes estuvieron cargo del contacto con posibles participantes. Se publicaron avisos en periódicos y redes sociales, y se ocuparon listas de correos institucionales para difundir el estudio y reclutar potenciales participantes. Luego de esto, se les proporcionó información sobre los objetivos y naturaleza del estudio, enfatizando la confidencialidad, el resguardo de la información y la participación voluntaria en la investigación. Previa firma del consentimiento informado, se procedió a la aplicación del cuestionario, bajo modalidad autoadministrada.

\section{Instrumentos}

Además de datos de caracterización sociodemográfica tales como sexo, edad, nivel educacional, y de caracterización del divorcio como iniciativa de la separación, tiempo desde la separación, se aplicó el Cuestionario de Perdón en el Divorcio-Separación (CPD-S, YárnozYaben \& Comino, 2012). Es una escala unidimensional, de auto-reporte, compuesta por 5 ítems en formato Likert (1=totalmente en desacuerdo, a 5=totalmente de acuerdo), cuyo objetivo es evaluar el perdón hacia la ex pareja. El instrumento posee un índice de consistencia interna de .77, medida mediante el alpha de Cronbach y asociación con variables como la satisfacción vital y la adaptación a la separación. En esta escala, puntajes más altos, indican mayores niveles de perdón.

The Abbreviated Scales of Depression, Anxiety and Stress (DASS-21, Lovibond, \& Lovibond, 1995), en su versión validada en Chile por Vinet, Rehbein, Román y Saiz (2008) (DASS21). Esta escala, compuesta por 21 items, mide la presencia de síntomas de depresión, ansiedad y estrés, en formato Likert de 0 (no describe nada de lo que me pasó o sentí durante la semana) a 3 (sí, esto me pasó mucho, o casi siempre). En el instrumento de Lovibond y Lovibond (1995), la consistencia interna basada en el alfa de Cronbach fue de .91 en la escala de depresión, 0.84 en la escala de ansiedad y 0.90 en la escala de estrés.

Escala de Satisfaccción con la Vida (Diener, Emmons, Larsen, \& Griffin, 1985). validada en Chile por Vera- Villarroel, Urzúa, Pavez, CelisAtenas y Silva (2012). Este instrumento mide el grado de satisfacción vital a partir de 5 reactivos, a los que se responde a través de una Escala Likert de 1 (no, en absoluto) a 7 puntos (si, totalmente). El índice de confiabilidad interna es de 0.82. Por otro lado, se obtuvo evidencia de su validez mediante su asociación con constructos teóricos relacionados. Para el presente estudio, la confiabilidad fue de .82 .

\section{Plan de análisis de datos}

De manera previa a los análisis, se examinaron los datos para evaluar el cumplimiento de los supuestos y el manejo de casos con elevados datos ausentes $(\mathrm{N}>10 \%)$ y atípicos. Luego de eso, se calcularon las medias, desviaciones típicas e índices de asimetría de los ítems.

Cabe mencionar que de acuerdo a lo establecido por las autoras de la escala original (Yárnoz-Yaben \& Comino, 2012), y de manera previa a los análisis, se procedió a la recodificación de los ítems inversos.

Con la muestra 1 se realizó un análisis paralelo (Horn, 1965) con la finalidad de determinar el número de factores, ocupando para ello el programa Factor 9.2 (Lorenzo-Seva \& Ferrando, 2013) y el SPSS versión 21. Este es uno de los métodos más recomendados para determinar el número de factores en el análisis factorial exploratorio (Ferrando \& Lorenzo-Seva, 2014; Hayton et al., 2004; Loret-Segura, FerreresTraver, Hernández-Baeza, \& Tomás-Marco, 2014).

Posteriormente, se llevó a cabo un Análisis Factorial Confirmatorio (AFC). Además del estadístico Chi cuadrado, se utilizaron los 
siguientes indicadores adicionales de ajuste, dada la sensibilidad de este al tamaño muestral: el Root Mean Square Error of Approximation (RMSEA) y su intervalo de confianza del 90\%, el Comparative Fit Index (CFI), el Tucker-Lewis Index (TLI), y el Standardized Root Mean Square Residual (SRMR). Se definieron como criterios de buen ajuste valores cercanos o menores a .08 en el caso del RMSEA valores sobre .95 en el CFI y el TLI, y menores a .06 en el SRMR. El análisis factorial confirmatorio se realizó con el programa MPLUS, versión 7.4 (Muthen \& Muthen, 2014) y Máxima Verosimilitud (ML) como método de estimación y la matriz de correlaciones de Pearson como input, dado que los valores de asimetría de los reactivos se encuentran por debajo de valores absolutos de +- 1.5. Además, se optó por tratar las variables como medidas de manera continua al ser una escala Likert de 5 puntos.

Posteriormente, se realizó además un análisis de invarianza métrica por sexo y tipo de relación (matrimonio y convivencia). La evaluación de la equivalencia del modelo unidimensional del CPD$\mathrm{S}$ según sexo y tipo de relación, se llevó a cabo mediante la comparación de modelos anidados, progresivamente más restrictivos. Para ello, se evaluaron tres tipos de invarianza: configural, en la que el número de factores, en este caso uno, y los patrones de cargas factoriales se estimaron libremente para ambos grupos; invarianza métrica, en la que se restringió a ser iguales las cargas factoriales; e invarianza escalar o métrica fuerte, en la que además se restringieron a ser iguales los interceptos. Para evaluar si estos modelos progresivamente más restrictivos difieren entre sí, se ocuparon como criterios la diferencia en el Chi cuadrado, así como los índices de ajuste comparativo propuestos por Millsap y Cham (2012) y Chen (2007): valores menores a .010 en el CFI, a .05 en el TLI y de .015 en el RMSEA se consideran como indicadores de equivalencia de los modelos.

Finalmente, se estimó la fiabilidad para el CPD-S mediante el cálculo del alfa de Cronbach y el alfa ordinal y se evaluó la asociación del CPD$\mathrm{S}$ con medidas de salud mental y satisfacción vital.

\section{Resultados}

\section{Análisis Factorial Exploratorio}

Los resultados del análisis paralelo realizado en la muestra 1 indican que el autovalor empírico del primer factor real (80.5), se encuentra por encima del promedio (63.9) y muy cercano al percentil 95 de los autovalores aleatorios (80.9). A partir del segundo factor, los autovalores empíricos se encuentran por debajo de la media y del percentil 95. Considerando estos resultados y ocupando el criterio más robusto, la solución más adecuada sería de un único factor. Se procedió entonces a realizar un análisis factorial exploratorio con dicha muestra ocupando máxima verosimilitud como método de estimación, el que arrojó una solución unifactorial como la más apropiada, con cargas significativas que fluctuaron entre .546 y .772 .

\section{Análisis Factorial Confirmatorio}

De manera previa a la realización del análisis factorial confirmatorio con la muestra 2 , se realizó un análisis de la estadística descriptiva de los ítems del CPD-S. En la Tabla 1 se presentan los promedios, desviaciones estándar e índices de asimetría y curtosis de cada uno de ellos. En ella se identifica que ninguno de los ítems tuvo una media o desviación extrema. Además, el análisis de la distribución de frecuencias de las opciones de respuesta revela que ningún ítem concentró más del $85 \%$ de las opciones extremas. En cuanto a los índices de asimetría estuvieron dentro de rangos aceptables, salvo el ítem 2, que supera levemente el valor absoluto de 1 .

Por otra parte la matriz de correlaciones de los ítems (ver Tabla 2), da cuenta de asociaciones significativas entre todos los ítems con valores fluctuantes entre .301 y .660 .

Con estos antecedentes, se examinó un primer modelo con ML como método de estimación, con un factor latente, de acuerdo a la propuesta original de las autoras, conteniendo 5 variables observadas. El modelo inicial evidenció un pobre ajuste a los datos, $\chi 2(5, N=715)=252.558, p<.001$, CFI $=.782$, TLI $=.565, \quad$ RMSEA $=.263,90 \%$ IC [.236, .291]; SRMR=.079. En términos del ajuste local, al examinar los pesos factoriales se observa 
Tabla 1. Medias, Desviaciones Estándar e Índices de Curtosis y Asimetría de los ítems del CPD-S

\begin{tabular}{lcccc}
\hline & M & DE & Curtosis Asimetría \\
\hline $\begin{array}{l}\text { 1. Estoy enojado/a con mi } \\
\text { ex pareja }\end{array}$ & 3.49 & 1.33 & -.95 & -.45 \\
$\begin{array}{l}\text { 2. No puedo evitar culpar } \\
\text { a mi ex pareja por haber } \\
\text { causado la ruptura }\end{array}$ & 3.46 & 1.29 & -1.03 & -.29 \\
$\begin{array}{l}\text { 3. He perdonado a mi ex } \\
\text { pareja }\end{array}$ & 3.31 & 1.21 & -.69 & -.33 \\
$\begin{array}{l}\text { 4. A pesar de que el } \\
\text { comportamiento de mi ex } \\
\text { - pareja me ha dolido, no }\end{array}$ & 3.51 & 1.25 & -.67 & -.54 \\
$\begin{array}{l}\text { le guardo rencor } \\
\begin{array}{l}\text { 5. Espero que mi ex } \\
\text { pareja reciba lo que se } \\
\text { merece por todo el daño }\end{array} \\
\text { que me hizo }\end{array}$ & & & & \\
\hline$N=715$ & & & & \\
\end{tabular}

Tabla 2. Matriz de correlaciones entre los ítems del CPD-S

\begin{tabular}{|c|c|c|c|c|c|}
\hline Item & 1 & 2 & 3 & 4 & 5 \\
\hline $\begin{array}{l}\text { 1. Estoy enojado/a con mi } \\
\text { ex pareja }\end{array}$ & i & & & & \\
\hline $\begin{array}{l}\text { 2. No puedo evitar culpar } \\
\text { a mi ex pareja por haber } \\
\text { causado la ruptura }\end{array}$ & $.594 * *$ & - & & & \\
\hline $\begin{array}{l}\text { 3. He perdonado a mi ex } \\
\text { pareja }\end{array}$ & $.444 * *$ & $321 * *$ & - & & \\
\hline $\begin{array}{l}\text { 4. A pesar de que el } \\
\text { comportamiento de mi ex } \\
\text { - pareja me ha dolido, no } \\
\text { le guardo rencor }\end{array}$ & $.341 * *$ & $301 * *$. & $660 * *$ & - & \\
\hline $\begin{array}{l}\text { 5. Espero que mi ex } \\
\text { pareja reciba lo que se } \\
\text { merece por todo el daño } \\
\text { que me hizo }\end{array}$ & $.458^{* *}$ & $460 * *$ & $375 * * 3$ & $349 * *$ & - \\
\hline
\end{tabular}

que todos los ítems tienen cargas significativas, con valores entre .611 y .721.

Pese al ajuste a nivel local, los indicadores de ajuste global indican que la estructura teórica hipotetizada no logra reproducir apropiadamente las relaciones observadas entre las variables.

Considerando el mal ajuste del modelo hipotetizado a los datos, y a partir del análisis de los residuos como de los índices de modificación sugeridos por el programa, se identificó la presencia de dos errores correlacionados de magnitud considerable, entre los ítems 3 y 4 ( $\mathrm{IM}=241.027)$.

Con base en estos resultados, se procedió a reestimar un segundo modelo, en el que se permitiese la correlación entre los errores de los ítems 3 y 4, dado que la revisión de ambos da cuenta de un traslape en sus contenidos.
Considerando que ambos reactivos corresponden a ítems en una misma dirección, es posible hipotetizar la presencia de un factor de método. Pese a esto, no fue posible especificar un factor de método como tal, dado que hacerlo implicaría problemas de identificación del modelo, concretamente, la ausencia de grados de libertad suficientes para evaluar el ajuste del modelo hipotetizado a los datos.

El ajuste de este segundo modelo fue aceptable, $\quad \chi 2(4, \quad \mathrm{~N}=715) \quad=26.360, \quad p<.001$, CFI $=.980, \quad$ TLI $=.951, \quad$ RMSEA $=.088,90 \%$ IC $[.058, .122]$; SRMR $=.029$. El valor del RMSEA se encuentra fuera de lo esperado, sin embargo existen antecedentes que este estadístico es menos eficiente ante muestras grandes o con parámetros elevados.

El examen de los pesos factoriales muestra que todos los ítems tienen cargas significativas y de magnitud apropiada (>.40) (ver Figura 1). El examen de los lambas de los ítems y de los porcentajes de varianza explicada, dan cuenta que el ítem 4 es el que explica el menor porcentaje de varianza del perdón a la ex pareja (18.8\%) y el ítem 1, el que evidencia el mayor porcentaje de varianza explicada (64\%).

Considerando estos resultados, se decidió retener el modelo 2 como el que mejor representa la estructura de los datos observados. En la Figura 1 se presentan las cargas factoriales estandarizadas obtenidas en el CPD-S.

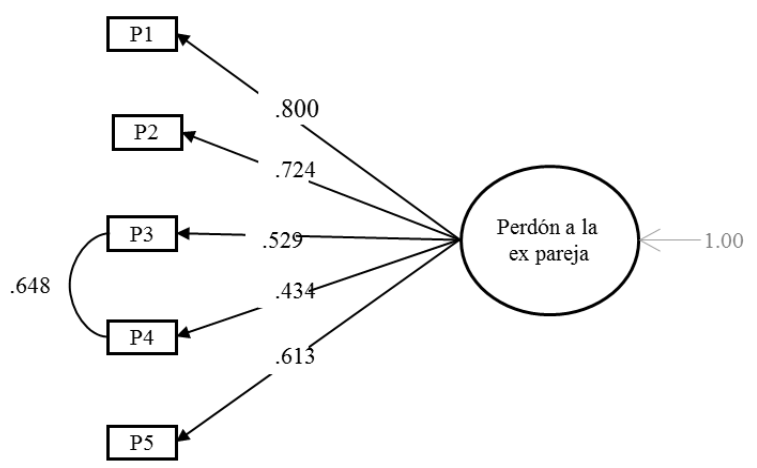

Figura 1. Cargas factoriales estandarizadas del modelo unidimensional del CPD-S con estimador ML

\section{Invarianza Métrica del CPD-S según Sexo y Tipo de Relación}

El modelo unifactorial evidenció un ajuste apropiado a los datos tanto para los hombres $\chi^{2}(4)=9.228, p<.01 ; \mathrm{CFI}=.99$; TLI=.974; RMSEA= 
Tabla 3. Índices de ajuste para Invarianza Métrica de la escala de perdón a la ex pareja según sexo

\begin{tabular}{lccccccccccc}
\hline & $\chi^{2}$ & $g l$ & CFI & TLI & RMSEA & $\Delta \chi^{2}$ & $\Delta \mathrm{gl}$ & $\Delta$ valor-p & $\Delta$ CFI & $\Delta$ TLI & $\Delta$ RMSEA \\
\hline Configural & 26.070 & 8 & .984 & .960 & .079 & & & & & & \\
Métrica & 39.887 & 12 & .975 & .959 & .081 & 13.817 & 4 & .04 & .009 & .001 & .002 \\
Escalar & 42.536 & 16 & .976 & .971 & .068 & 2.65 & 4 & .61 & .001 & .012 & .013 \\
Nota . Mujeres $=358 ;$ Hombres $=357$ & & & & & & & & &
\end{tabular}

Tabla 4. Índices de Ajuste para Invarianza Métrica del Cuestionario de Perdón a la ex pareja según Tipo de Relación

\begin{tabular}{lccccccccccc}
\hline & $\chi^{2}$ & $g l$ & CFI & TLI & RMSEA & $\Delta \chi^{2}$ & $\Delta \mathrm{gl}$ & $\Delta$ valor-p & $\Delta$ CFI & $\Delta$ TLI & $\Delta$ RMSEA \\
\hline Configural & 28.166 & 8 & .982 & .955 & .084 & & & & & & \\
Métrica & 34.871 & 12 & .980 & .966 & .073 & 6.705 & 4 & .28 & .002 & .011 & .011 \\
Escalar & 37.829 & 16 & .981 & .976 & .062 & 2.948 & 4 & .56 & .001 & .010 & .011 \\
\hline
\end{tabular}

Nota. Matrimonio $=405 ;$ Convivencia $=310$

.061 [90\% CI: .000-.113]; SRMR=.028, como para las mujeres, $\chi^{2} \quad(4)=16.842, \quad p<.01$; CFI $=.980 ;$ TLI $=.949 ; \quad$ RMSEA $=.095$ [90\% CI: $.051-.143 ;$ SRMR=.030.

La evaluación de la invarianza según sexo presentada en la Tabla 1, arroja una diferencia significativa entre el modelo configural y el métrico $\Delta \chi^{2}=13.82, \Delta \mathrm{gl}=4, p=.04$, al comparar la diferencia en el chi cuadrado. Sin embargo, las diferencias en los índices de ajuste incremental están por debajo de los criterios establecidos para señalar sostener la no equivalencia, por lo que puede sostenerse que la estructura del CPD-S es similar para hombres y mujeres.

Luego, al comparar el modelo con las cargas factoriales restringidas a ser iguales con el más restrictivo que asume igualdad en los interceptos, los resultados revelan que el ajuste no empeora significativamente, $\Delta \chi^{2}=2.65, \Delta \mathrm{gl}=4, p=.61$ al comparar los valores de Chi cuadrado y al examinar las diferencias en los otros indicadores de ajuste. Por ende, es posible afirmar que el CPD-S funciona de manera similar en hombres y mujeres.

Respecto de la comparación entre personas separadas provenientes de matrimonios y uniones de hecho, el análisis del ajuste del modelo hipotetizado por separado para ambos grupos, da cuenta de un ajuste aceptable a los datos, tanto para el grupo de personas provenientes de convivencia $\chi^{2}(4)=15.182, \quad p<.01 ; \quad$ CFI=.974; TLI=.935; RMSEA=.095 [90\% CI: .047-.148]; $\mathrm{SRMR}=.035$, como para quienes provienen de matrimonios, $\chi^{2}(4)=12.985, p<.05 ; \mathrm{CFI}=.987$; TLI=.967; RMSEA=.074 [90\% CI: .032- .121; SRMR=.026.

Luego, los resultados presentados en la Tabla 3, dan cuenta de la presencia de invarianza configural, métrica y escalar, por lo que la estructura factorial CPD-S es equivalente entre las personas separadas provenientes de relaciones de matrimonio como de convivencias.

\section{Consistencia Interna}

La fiabilidad de la escala estimada mediante el alfa de Cronbach fue de .79 y de .75 mediante el alfa ordinal, valores considerados aceptables tomando en cuenta la reducida cantidad de ítems de la escala.

\section{Validez convergente}

De acuerdo a lo observado en la Tabla 4, el perdón a la ex pareja se asocia con mayores niveles de satisfacción con la vida y a menores niveles de ansiedad, depresión y estrés.

Tabla 5. Correlación entre el CPDS-CH, la Satisfacción Vital e índices de Salud mental

\begin{tabular}{lccccc}
\hline \multicolumn{1}{c}{ Perdón a la } & 1 & 2 & 3 & 4 & 5 \\
\hline $\begin{array}{l}\text { ex pareja } \\
\text { 2.Satisfacción }\end{array}$ & 1 & & & \\
vital & $.22 * *$ & 1 & & \\
3. Ansiedad & $-.28^{* *}$ & $-.33^{* *}$ & 1 & \\
4. Depresión & $-.26 * *$ & $-.22^{* *}$ & $.80^{*}$ & 1 \\
5. Estrés & $-.28^{* *}$ & $-.27 * *$ & $.81^{* *}$ & $.80^{* *}$ \\
\hline$p \leq .05$. & & & &
\end{tabular}




\section{Conclusiones}

El objetivo de este estudio fue evaluar la estructura factorial e invarianza métrica según sexo y tipo de relación de las puntuaciones del CPD-S en contexto chileno, así como examinar su consistencia interna y su asociación con constructos teóricamente relacionados.

De los análisis realizados, se puede concluir que el modelo unidimensional representa aceptablemente los datos observados.

Pese a ello, es importante notar que se identificó la existencia de dos errores correlacionados cuyo significado no pudo ser explorado, porque hacerlo trae consigo problemas de identificación del modelo. Una posible explicación para este resultado, guarda relación con que tales ítems, que son los que tienen una dirección diferente a los restantes, correspondan a un factor de método. Una explicación alternativa, de carácter más sustantivo, podría tener que ver con que hay autores que han postulado que el perdón, en algunos casos tendría un carácter bidimensional; esto es, que involucraría no solo la disminución del resentimiento y la rabia hacia el otro (dimensión negativa), sino también el desarrollo de sentimientos positivos hacían quien cometió la ofensa (dimensión positiva) (e.g., Kato, 2015; Rye et al., 2001). Cabe señalar, no obstante, que la bidimensionalidad de este constructo se ha propuesto particularmente para el perdón que ocurre en relaciones cercanas que se mantienen (por ejemplo, en relaciones de pareja). La pequeña cantidad de reactivos que componen esta escala no permitieron poner a prueba dicha hipótesis, dada la pérdida de grados de libertad asociados a la especificación de un factor adicional y a que en tal caso, dicho factor hubiese quedado conformado por solo dos reactivos. Futuros estudios podrían eventualmente evaluar una versión revisada de la escala, añadiendo quizás ítems y probar nuevamente la configuración de la misma.

Por otra parte, se identificó que la estructura factorial del CPD-S es invariante según género y tipo de relación (matrimonios y uniones de hecho), aportando con ello información adicional sobre este instrumento, dado que la equivalencia del CPD-S no había sido testeada previamente. Es decir, los ítems cargan en un único factor en cada grupo, los pesos factoriales son de similar magnitud y los interceptos son similares entre los grupos. Por lo tanto, se puede concluir que el CPD-S es equivalente por género y tipo de relación. De esta manera, es posible sostener que las posibles comparaciones entre dichos grupos son válidas, puesto que las puntuaciones del CPD$\mathrm{S}$ tienen el mismo significado entre hombres y mujeres por un lado, y entre personas provenientes de matrimonios y uniones de hecho por otro.

Adicionalmente y de acuerdo a lo esperado, el perdón a la ex pareja se asoció a menores problemas de salud mental y a mayores índices de salud mental, lo cual es coherente con antecedentes previos que documentan los efectos beneficiosos del perdón (e.g., Rohde-Brown et al., 2011; Rye et al., 2004; Yárnoz-Yaben, 2013; Yárnoz-Yaben, Garmendia, \& Comino, 2016).

Las limitaciones del estudio incluyen la necesidad de tener cautela respecto de la generalización de los resultados, dado el carácter no probabilístico del muestreo y a una menor representación de personas con menor nivel de escolaridad, debido a que la muestra estuvo compuesta principalmente por personas con estudios técnicos o universitarios. Futuros estudios podrían subsanar estas limitaciones evaluando el comportamiento particularmente de la CPD-S en una muestra con características más heterogéneas.

Desde un punto de vista clínico, contar con un instrumento de este tipo en el contexto chileno, tiene utilidad para el trabajo con personas y familias en proceso de separación, pues otorga una herramienta para evaluar cómo se encuentran las personas que solicitan ayuda psicoterapéutica en aspectos específicos que se relacionan con la adaptación al divorcio y la separación, como lo es el perdón. El trabajo con el dolor por las heridas ocurridas durante la relación y la ruptura misma a través del perdón como una estrategia dentro de otras posibles, es un tema frecuente en el ámbito psicoterapéutico.

Finalmente y a modo de conclusión, el CPD-S representa el inicio de un cuerpo de investigación en el ámbito del divorcio y/o separación en Chile, desde la perspectiva de la psicología clínica, dado que los estudios disponibles a la fecha han sido abordados desde una mirada predominantemente 
jurídica (Cox, 2011) y/o sociológica (Gómez, 2012).

\section{Referencias}

Amato, P. R. (2010). Research on divorce: Continuing trends and new developments. Journal of Marriage and Family, 72(3), 650666. doi:10.1111/j.1741-3737.2010.00723.x

Berrington, A., Perelli-Harris, B., \& Trevena, P. (2015). Commitment and the changing sequence of cohabitation, childbearing, and marriage: Insights from qualitative research in the UK. Demographic Research, 33(12), 327362. doi:10.4054/demres.2015.33.12

Berry, J. W., Worthington, E. L., Parrott, L., O'Connor, L. E., \& Wade, N. G. (2001). Dispositional forgivingness: Development and construct validity of the Transgression Narrative Test of Forgivingness (TNTF). Personality and Social Psychology Bulletin, 27(10), 1277-1290. doi:10.1177/01461672012710004

Bonach, K. (2007). Forgiveness intervention model. Journal of Divorce \& Remarriage, 48(1-2), 105-123. doi:10.1300/j087v48n01_06

Bonach, K. (2009). Empirical support for the application of the forgiveness intervention model to postdivorce coparenting. Journal of Divorce \& Remarriage, 50(1), 38-54. doi:10.1080/10502550802365631

Bonach, K., \& Sales, E. (2002). Forgiveness as a mediator between post divorce cognitive processes and coparenting quality. Journal of Divorce \& Remarriage, 38(1-2), 17-38. doi:10.1300/j087v38n01_02

Cáceres C., Manhey C., \& Raies A. (2004). Comprensión sistémico-relacional del proceso de separación conyugal. Revista de Familias y Terapias, 12(18), 31-54.

Chen, F. F. (2007). Sensitivity of goodness of fit indexes to lack of measurement invariance. Structural Equation Modeling: A Multidisciplinary Journal, 14(3), 464-504. doi:10.1080/10705510701301834

Cox, L. (2011). Divorcio en Chile. Un análisis preliminar tras la nueva ley de matrimonio civil. Estudios Públicos, 123, 95-187.

Diener, E., Emmons, R. A., Larsen, R. J., \& Griffin, S. (1985). The Satisfaction With Life
Scale. Journal of Personality Assessment, 49(1), 71-75. doi:10.1207/s15327752jpa4901_13

Fabricius, W. V., \& Luecken, L. J. (2007). Postdivorce living arrangements, parent conflict, and long-term physical health correlates for children of divorce. Journal of Family Psychology, 21(2), 195-205. doi:10.1037/0893-3200.21.2.195

Ferrando, P. J., \& Lorenzo-Seva, U. (2014). El análisis factorial exploratorio de los ítems: Algunas consideraciones adicionales. Anales de Psicología, 30(3), 1170-1175.

Freedman, S., \& Knupp, A. (2003). The impact of forgiveness on adolescent adjustment to parental divorce. Journal of Divorce \& Remarriage, 39(1-2), 135-165. doi:10.1300/j087v39n01_08

Gómez, V. (2012). Divórcio à la chilena: Familia, gênero e cidadania no Chile, 1990-2004. Revista de Sociología e Política, 20(44), 177195. doi:10.1590/s0104-44782012000400013

Gordon, K. C., \& Baucom, D. H. (2003). Forgiveness and marriage: Preliminary support for a measure based on a model of recovery from a marital betrayal. The American Journal of Family Therapy, 31(3), 179-199. doi:10.1080/01926180301115

Guzmán, M. (2010). El perdón en relaciones cercanas: Conceptualización desde una perspectiva psicológica e implicancias para la práctica clínica. Psykhe, 19(1), 19-30.

doi:10.4067/S0718-22282010000100002

Hambleton, R. K., Merenda, P. F., \& Spielberg, C. D. (2005). Adapting educational and psychological test for cross-cultural assessment. Mahwah, NJ: Lawrence Erlbaum.

Hayton, J. C., Allen, D. G. y Scarpello, V. (2004). Factor retention decisions in exploratory factor analysis: A tutorial on parallel analysis. Organizational Research Methods, 7, 191205.

Horn, J. L. (1965). A rationale and test for the number of factors in factor analysis. Psychometrika, 30(2), 179-185. doi:10.1007/bf02289447

Kato, T. (2015). Effects of forgiveness for a partner on psychological dysfunction in dating relationship among freethinkers: A longitudinal study. In E. L. Olsen (Ed.), Forgiveness: Social significance, health 
impact and psychological effects (pp. 61-82). Hauppauge, NY: NOVA Science Publishers.

Lebow, J., \& Newcombrekart, K. (2007). Integrative family therapy for high-conflict divorce with disputes over child custody and visitation. Family Process, 46(1), 79-91. doi:10.1111/j.1545-5300.2006.00193.x

Lloret-Segura, S., Ferreres-Traver, A., HernándezBaeza, A., \& Tomás-Marco, I. (2014). El análisis factorial exploratorio de los ítems: Una guía práctica, revisada y actualizada. Anales de Psicología, 30(3), 1151-1169.

Lorenzo-Seva, U., \& Ferrando, P. J. (2013). FACTOR 9.2: A Comprehensive Program for Fitting Exploratory and Semiconfirmatory Factor Analysis and IRT Models. Applied Psychological Measurement, 37(6), 497-498. doi:10.1177/0146621613487794

Lovibond, P., \& Lovibond, S. (1995). The structure of negative emotional states: Comparison of the Depression Anxiety Stress Scales (DASS) with the Beck depression and anxiety inventories. Behavior Research and Therapy, 33(3), 335-343. doi:10.1016/0005-7967(94) 00075-u

McCullough, M. E., Rachal, K. C., Sandage, S. J., Worthington, E. L., Brown, S. W., \& Hight, T. L. (1998). Transgression-Related Interpersonal Motivations Inventory. PsycTESTS Dataset. doi:10.1037/t03960-000

McCullough, M. E., Root, L. M., \& Cohen, A. D. (2006). Writing about the benefits of an interpersonal transgression facilitates forgiveness. Journal of Consulting and Clinical Psychology, 74(5), 887-897. doi:10.1037/0022-006x.74.5.887

Meyers, L. S., Gamst, G., \& Guarino, A. J. (2013). Applied Multivariate Research. Sage: Thousands Oaks

Millsap, R. E., \& Cham, H. (2012). Investigating factorial invariance in longitudinal research. In B. Laursen, T. D. Little \& N. A. Card (Eds.), Handbook of developmental research methods (pp. 109-126). New York, NY: Guilford Press.

Muthén, L. K., \& Muthén, B. O. (2014). Mplus user's guide (7th ed.). Los Angeles, CA: Authors.

Ojeda García, A., Torres González, T., \& Moreira Mayo, M. (2010). ¿Amor y compromiso en la pareja?: De la teoría a la práctica. Revista Iberoamericana de Diagnóstico y Evaluación - e Avaliação Psicológica, 2(30), 125-142.

Paleari, F. G., Regalia, C., \& Fincham, F. D. (2009). Measuring offence-specific forgiveness in marriage: The Marital OffenceSpecific Forgiveness Scale (MOFS). Psychological Assessment, 21(2), 194-209. doi:10.1037/a0016068

Rohde-Brown, J., \& Rudestam, K. E. (2011). The role of forgiveness in divorce adjustment and the impact of affect. Journal of Divorce \& Remarriage, 52(2), 109-124. doi:10.1080/10502556.2011.546233

Rye, M. S., Fleri, A. M., Moore, C. D., Worthington, E. L., Wade, N. G., Sandage, S. J., \& Cook, K. M. (2012). Evaluation of an intervention designed to help divorced parents forgive their ex-spouse. Journal of Divorce \& Remarriage, 53(3), 231-245. doi:10.1080/10502556.2012.663275

Rye, M. S., Folck, C. D., Heim, T. A., Olszewski, B. T., \& Traina, E. (2004). Forgiveness of an ex-spouse. Journal of Divorce \& Remarriage, 41(3-4), 31-51. doi:10.1300/j087v41n03_02

Rye, M. S., Loiacono, D. M., Folck, C. D., Olszewski, B. T., Heim, T. A., \& Madia, B. P. (2001). Evaluation of the psychometric properties of two forgiveness scales. Current Psychology, 20(3), 260-277. doi:10.1007/s12144-001-1011-6

Sánchez - Aragón, R., \& Retana - Franco, B. (2013). Evaluación tridimensional del duelo amoroso en México. Revista Iberoamericana de Diagnóstico y Evaluación - e Avaliação Psicológica, 36(2), 49-69.

Sbarra, D. A. (2015). Divorce and health. Psychosomatic Medicine, 77(3), 227-236. doi:10.1097/psy.0000000000000168

Sbarra, D. A., Hasselmo, K., \& Bourassa, K. J. (2015). Divorce and health. Current Directions in Psychological Science, 24(2), 109-113. doi:10.1177/0963721414559125

Strelan, P., \& Covic, T. (2006). A review of forgiveness process models and a coping framework to guide future research. Journal of Social and Clinical Psychology, 25(10), 1059-1085. doi:10.1521/jscp.2006.25.10.1059

Subkoviak, M. J., Enright, R. D., Wu, C.-R., Gassin, E. A., Freedman, S., Olson, L. M., \& 
Sarinopoulos, I. (1995). Measuring interpersonal forgiveness in late adolescence and middle adulthood. Journal of Adolescence, $\quad$ 641-655), doi:10.1006/jado.1995.1045

Svensson, M., \& Siles, C. (2014). Vivir juntos, reflexiones sobre la convivencia en Chile. Instituto de Estudios de la Sociedad. Cuadernillos IES: Chile.

Sweeper, S., \& Halford, K. (2006). Assessing adult adjustment to relationship separation: The Psychological Adjustment to Separation Test (PAST). Journal of Family Psychology, 20(4), 632-640. doi:10.1037/0893-3200.20.4.632

Tashiro, T., Frazier, P., \& Berman, M. (2006). Stress-related growth following divorce and relationship dissolution. En M. Fine \& J. Harvey (Eds.), Handbook of divorce and relationship dissolution (pp. 361-384). New York: Routledge Taylor \& Francis Group.

Thompson, L. Y., Snyder, C. R., Hoffman, L., Michael, S. T., Rasmussen, H. N., Billings, L. S., ... Roberts, D. E. (2005). Dispositional forgiveness of self, others, and situations. Journal of Personality, 73, 313-360. doi:10.1111/j.1467-6494.2005.00311.x

Trinder, L., Kellet, J., \& Swift, L. (2008). The relationship between contact and child adjustment in high conflict cases after divorce or separation. Child and Adolescent Mental Health, 13(4), 181-187. doi:10.111/j.1475-3588.2008.00484.x

United Nations (2011). 2009-2010. Demographic Yearbook. Department of economic and social affairs. New York: Author.

Vera-Villarroel, P., Urzúa, M. A., Pavez, P., Celis-Atenas, K., \& Silva, J. (2012). Evaluation of subjective well-being: Analysis of the Satisfaction with Life Scale in Chilean population. Universitas Psychologica, 11 (3), 719-727.

Vinet, E., Rehbein, L., Román F., \& Saiz, J. (2008). Escalas abreviadas de depresión, ansiedad y estrés (DASS-21). Versión chilena traducida y adaptada. Documento no publicado, Universidad de La Frontera, Temuco, Chile.

Yárnoz Yaben, S., Garmendia, A., \& Comino, P. (2016). Looking at the bright side: Forgiveness and subjective well-being in divorced Spanish parents. Journal of Happiness Studies, 17, 1905-1919. doi:10.1007/s10902-015-9677-x

Yárnoz-Yaben, S. (2013). Forgiveness, adjustment to divorce and support from the former spouse in Spain. Journal of Child and Family Studies, 24(2), 289-297. doi:10.1007/s10826-013-9835-X

Yárnoz-Yaben, S., \& Comino, P. (2012). Un instrumento para la evaluación del perdón en el ámbito del divorcio y la separación. International Journal of Psychology and Psychological Therapy, 12(1), 49-58. 